\title{
Performance Comparison of Adaptive and Blind Equalization Algorithms for Wireless Communication
}

\author{
K. Suthendran and T. Arivoli
}

\begin{abstract}
Adaptive equalization is a well known method to minimize the Inter-Symbol Interference (ISI) in wireless communication. Often, adaptive algorithm requires transmission of known training sequence to track the time varying characteristics of the channel and hence utilizes additional bandwidth. It is also impractical to have training sequences in all types of transmissions (e.g. non-cooperative environment). Blind algorithm is a concept to track the time varying characteristics of the channel in the absence of training sequence. However, it leads to slow convergence. In this paper, we compare the performance of adaptive LMS algorithm and SATO based blind algorithm for PAM signal.
\end{abstract}

Keywords---Adaptive Equalization, Blind Equalization, Convergence, LMS algorithm, SATO Algorithm

\section{INTRODUCTION}

$\mathrm{I}$ $\mathrm{n}$ the trendy electronic communication, plenty of effort has been dedicated to utilize the accessible channel bandwidthexpeditiously. Intersymbol Interference (ISI) and Thermal noise are the two main factors that are limiting the performance of information transmission systems. In essence, the ISI is generated by dispersion within the transmit filter, the transmission medium, and receive filter. Within the bandlimited (frequency selective) time dispersive channel, the ISI is caused by multipath propagation. The result is that the modulated pulses are unfolded in time into adjacent symbols, and it distorts the transmitted signals inflicting information errors at the receiver. Thermal noise is generated at the face of the receiver. For bandwidth-limited channels, the ISI has been recognized as the major downside in high speed information transmission over wireless channels. The standard band restricted filters fail to recover the information once the received symbol contains ISI and in-band noise. The Intersymbol Interference will be removed by victimization equalization techniques. Sato has proposed 0.0006 to be the optimum step size value for blind equalization having a PAM signal [1].The authors in [12] have stated that the convergence

K. Suthendran, Assistant Professor, Department of Electronics and Communication Engineering, Kalasalingam Academy of Research andEducation, Krishnankoi-626 126, India. Email:rksuthendranster@gmail.com

T. Arivoli, Professor \& Head, Department of Electronics and Communication Engineering, Vickram College of Engineering Enathi-630 56,India.E-mail:t.arivoli@gmail.com

DOI: 10.9756/BIJRCE.10063 speed with the adaptive LMS algorithm is to be better for a given step size of 0.015 . But our experimental results show that $\alpha$ of 0.0006 is simply too low and thus leads to slow convergence. Whereas $\alpha$ value of 0.6 is the optimum one that supports quicker convergence and beyond this value itdoes not converge for any PAM symbol. In section IV we have compared the performance of adaptive and blind equalization algorithms for a PAM input signal.

\section{ADAPTIVE EQUALIZERS}

Generally, the term equalization is employed to explain any signal process operation that minimizes the ISI [8]. DSP-based equalizer systems become essential in various applications including information, voice, and video communications. The equalizer may be a digital filter, placed between sampler and decision algorithm within theband restricted communication

model. An equalizer inside the receiver compensates for average range of expected channel amplitude and delay characteristics. Equalizer algorithmic program, equalizer structure and therate of amendment of the multipath radio channel are three main factors that have an effect on the time spread over that an equalizer converges. Two important issues inequalizer design and

implementation are its complexnessand its training. For frequency selective channel, the equalizer enhances the frequency parts with tiny amplitudes and attenuates the robust frequencies within the received frequency response and for a time-varying channel.

An equalizer corrects the channel frequency response variation and cancels the multipath weakening effects. They're specifically designed for multipath correction and are therefore usually termed as echo-cancellers or deghosters [9]. For this it's going to need considerably longer filter length than that of easy spectral equalizers; however the principles of operation are basically constant. These filters have an equalized impulse response having zero ISI and zero channel distortion. This implies that convolution of the channel and the equalizer impulse responses should be equal, having one at the centre tap and nulls at the opposite sample points inside the filter span [7], [9] and [10].

Automatic synthesis and adaptation are the two strategies, used to estimate the filter coefficients. In automatic synthesis methodology, the equalizer generally compares a received time-domain reference signal thereto of an ingenuous training signal. This is often holding on within the receiver and a timedomain error signal is decided. The calculated error signal is 
employed to estimate the inverse filter coefficient. In an adaptation filter synthesis methodology, the equalizer calculates the error signal supported the distinction between the output of the equalizer and therefore the calculable transmitted signal that is generated by a decision device. The filter coefficient valuesarechangedfor every iterationcorresponding to the error signal value and, they're optimized with zero error. The main disadvantage during this automatic synthesis equalization methodology is that whereas sending a training signal the overhead related to it, ought to at least have the length of the filter tap. This needs training, in serving to the filter to converge at the startup that could be a part of the initialization overhead.

The mobile weakening channel may be a random and time varying; equalizers should track the time varying characteristics of the channel, and therefore known as adaptive equalizers. Adaptive channel equalization is a good tool in mitigating inter-symbol interference (ISI) caused by linear distortions in unknown channels [5]. An adaptive filtering formula needs the data regarding the "known" response therefore on estimate the error signal required for adaptive method. In apply; the better-known signal may be generated at the receiver facet in 2 ways. In first technique, the transmitted training sequence is retrieved by the reproduction of the better-known response that is keep within the receiver. The synchronization ought to be done between transmitted training sequence and better-known response that is keep within the receiver. With a better-known training sequence, the adaptive filtering formula used to modify the equalizer coefficients, which corresponds mathematically to finding out the distinctive minimum quadratic error performance surface. The second technique may be a decision directed technique, in which, rather than the better-known training sequence, a sequence of information symbols are calculable from the equalizer may be used. This calculable output is also unreliable; therefore this might not permit the tap weight coefficients to be optimized.

In general, the computation of error estimation is completed with the aid of the input vector and desired response, and it's accustomed create the control over the adjustable filter coefficients values. betting on the filter structure chosen, the adjustable coefficients is also in style of tap weight reflection coefficients, or rotation parameters. However, the elemental distinction between the assorted applications of adaptive filtering arises within the manner during which the required response is extracted.

Training and tracking are the two general operational modes of an adaptive equalizer. First, a legendary training sequence -pseudorandom binary signal of fixed length is shipped by the transmitter. With this, the equalizer at the receiver facet could adapt to a correct weight for minimum bit error rate (BER) detection. Following this training sequence, original information is shipped and adaptive equalizer utilizes the recursive formula to gauge the channel, and therefore estimates the filter coefficients to compensate the distortion created by multipath within the channel. Equalizers need periodic preparation so as to keep up effective ISI cancellation. In digital communication systems, user information is generally segmented into short time blocks or time slots. Time division multiple access (TDMA) wireless systems are notably compatible for equalizers. Owing to time variable nature of wireless channels, training signals should be sent often and this occupies additional information measure. In several applications legendary training sequence is needed to adapt the equalizers by minimizing the mean square error [MSE], however this being impractical and expensive once long training sequence is important [7]. As an example, in step with $900 \mathrm{MHz}$ GSM customary, twenty six bits out of each 148 bit frame are used as training signals [3] and [11].

\section{A. Least Mean Square Algorithm}

In this section we tend to mentioned regarding the standard Least Mean square (LMS) adaptive equalizer algorithm Figure. 1 shows the structure of an adaptive least mean square algorithm that encompasses the matched filtering action. The final word aim of this algorithm is to regulate the equalizer tap coefficients with the reference of the required response.

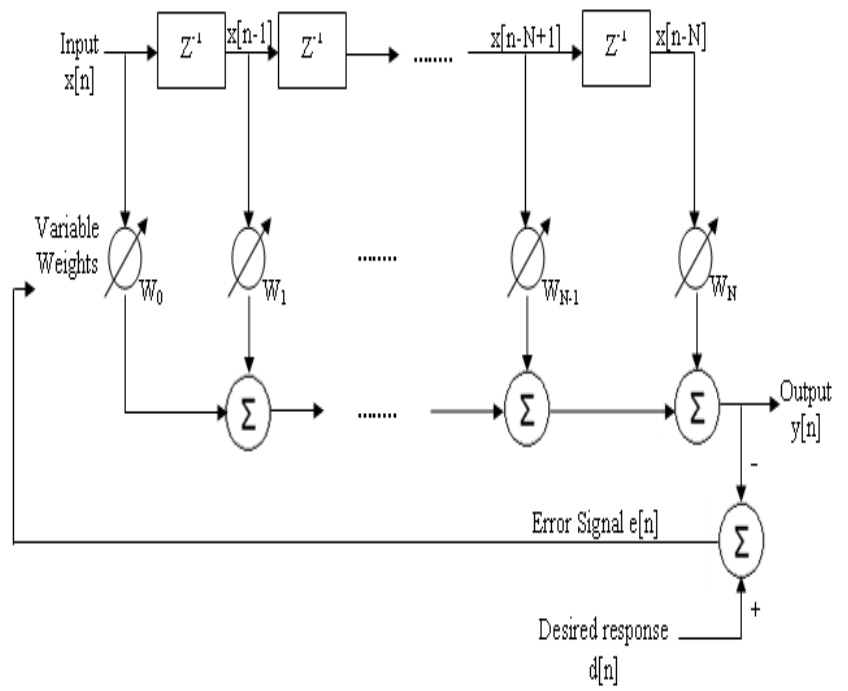

Figure 1: The LMS adaptive equalizer with 5 taps

The output $y[n]$ of the tapped delay line equalizer corresponding to the input sequence $\{x[n]\}$ is outlined by the discrete convolution total,

$$
y[n]=\sum_{k=0}^{N} w_{k} x[n-k]
$$

Where $w_{k}$ is that the $k^{\text {th }}$ tap weight, $N+1$ is that the total range of taps present within the equalizer.

The tap weight represents the adaptive filter coefficients. At each sampling instant, the error is determined by comparing the specified response that is understood at the receiver and therefore the actual response. With the assistance of obtained error, the filter will estimate the direction during which the tap weights ought to be modified and therefore the adaptation is also achieved within the adaptive filter. Let $\mathrm{a}[\mathrm{n}]$ denote the known response of nth transmitted binary symbol. The distinction between the specified response $\mathrm{a}[\mathrm{n}]$ and therefore the actual response $y[n]$ of the equalizer denote the error signal $e[n]$, and expressed as $e[n]=a[n]-y[n]$.

In LMS algorithm, individual tap weights change to each 
iteration are controlled by the obtained error value e[n]. LMS algorithm is expressed as follows.

$\left(\begin{array}{l}\text { Updated } \\ \text { value of } \\ \mathrm{k}^{\text {th }} \text { tap } \\ \text { weight }\end{array}\right)=\left(\begin{array}{l}\text { Old value } \\ \text { of } \\ \mathrm{k}^{\text {th }} \text { tap } \\ \text { weight }\end{array}\right)+\left(\begin{array}{l}\text { Step size } \\ \text { parameter }\end{array}\right) *\left(\begin{array}{l}\text { Input signal } \\ \text { applied to } \\ \mathrm{k}^{\text {th }} \text { tap } \\ \text { weight }\end{array}\right) *\left(\begin{array}{l}\text { Error } \\ \text { signal }\end{array}\right)$

Let $\mu$ denote the step size parameter of the filter. From the figure.1, the input signal given to the $k^{\text {th }}$ tap weight at time step $n$ is $x[n-k]$. Hence, using $\hat{w}_{k}(n)$ as the previous value of the $\mathrm{k}^{\text {th }}$ tap weight at time step $\mathrm{n}$, the updated value of this tap weight at time step $n+1$ is calculated from the equation (2), defined by,

$$
\begin{gathered}
\hat{w_{k}}[n+1]=\hat{w_{k}}[n]+\mu \cdot x[n-k] \cdot e[n] \\
k=0,1, \ldots ., N
\end{gathered}
$$

Where,

$$
e[n]=a[n]-\sum_{k=0}^{N} \hat{w}_{k}[n] \cdot x[n-k]
$$

The adaptive least mean square algorithm represented by equation (2) and (3).

\section{BLIND EQUALIZERS}

Even though trained strategies have many disadvantages, they're typically adequate. The throughput of the system drops owing to the time slots occupied by the training signal. Another disadvantage is that the training signal isn't forever familiar at the receiver, e.g., in an exceedingly non cooperative (surveillance) surroundings. Finally, the quicker time variable channel needs training sequence a more often to train the equalizer. This results in more reduction within the throughput of the system.

The Blind algorithms are ready to exploit characteristics of the transmitted signals and don't need training sequences. They're called so because they supply equalizer convergence without burdening the transmitter with training overhead. These fashionable algorithms are able to acquire equalization through property restoral techniques of the transmitted signal. In general, even once the initial error rate is massive, blind equalization technique directs the coefficient adaptation method towards the optimum filter parameters. A Blind Equalizer is in a position to compensate amplitude and delay distortions of a communication channel using solely the channel output samples and also the data of the basic statistical properties of the information symbols. the key advantage of blind equalizers is that there's no training sequence to calculate the tap weight coefficients; thus no bandwidth is wasted by its transmission. Blind equalization is effective for a high-speed digital radio, digital mobile communication systems, multi-point networks, cable TV, and digital terrestrial TV broadcasting [6] and [10].

The major downside is that the equalizer can usually take a extended time to converge as compared to a trained equalizer.
The necessity for blind equalizers within the field of information communications is greatly mentioned by Godard [2], within the context of multipoint networks. Blind joint

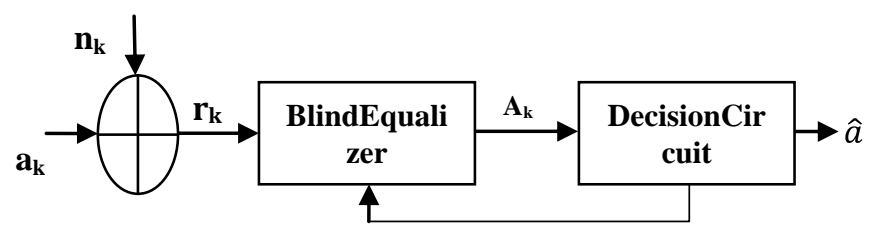

Figure 2: General block diagram for Blind equalizer equalization and carrier recovery might realize application in digital communication system over multipath weakening channels. Moreover, it's applied in extremely non-stationary digital mobile communications, wherever it's impractical to use training sequences. These techniques embrace algorithms like the SATO algorithm and Constant modulus algorithm (CMA).

\section{A. Sato's blind algorithm}

Sato was the one who first introduced the concept of blind equalization in 1975 for multilevel pulse amplitude modulation, wherever there's no reference sequence accessible and afterward Godard combined Sato's idea with a decision Directed (DD) algorithm and acquire a replacement blind equalization scheme for QAM data transmission. Since blind equalization has attracted significant scientific interest due to its potentials in terms of overhead reduction and simplification of point to multipoint communication. Sato proposed algorithm that was designed just for real valued signal and PAM [1]. However, its advanced valued extension is simple, that was derived by Godard [2] and [13]. The cost function proposed by SATO is given in (4),

$$
\text { sato }(A)=E\left\{\left(y_{k}-\gamma \cdot \operatorname{sign}\left(y_{k}\right)^{2}\right)\right\}
$$

Where,

$$
\begin{aligned}
& y_{k}=\text { output of the equalizer } \\
& \operatorname{sign}\left(y_{k}\right)=\left\{\begin{array}{l}
1, y_{k}>0 \\
-1, y_{k}<0
\end{array}\right. \\
& \gamma=\frac{E\left(a_{k}^{2}\right)}{\left|E\left(a_{k}\right)\right|}
\end{aligned}
$$

Sign denotes the standard signum function of a real scalar. $\gamma$ referred as scaling factor and $a_{k}$ denotes the input data sequence.

Figure. 2 show the general block diagram of the Blind Equalizer. It seems that Sato's proposal appears to be developed over LMS algorithm that uses steepest decent criteria for convergence process. Mathematically, if we tend to differentiate any equation and equate it to zero, then this provides the minimum; substituting it to the steepest-descent criteria, we are going to get the tap weight coefficients for the equalizer. If we tend to differentiate (4) and substitute it to the 
steepest-descent criteria, we are going to be obtaining (7) as shown. The algorithm of SATO's blind equalization relies on (7), that is employed for training the output sequences,

$$
\hat{A}_{k+1}=\hat{A}_{k}-\alpha \cdot r_{k}\left[y_{k}-\gamma \cdot \operatorname{sign}\left(y_{k}\right)\right]
$$

Where,

$$
\begin{array}{lll}
\mathrm{A}_{k} & = & \text { Weight used for training } \\
\mathrm{A} & = & \text { Tap-adjusting coefficient } \\
\mathrm{y}_{k} & = & \text { Output sequence } \\
\mathrm{r}_{k} & = & \text { Input sequence } \\
& r_{k}=\sum_{i=-\infty}^{\infty} a_{i} \cdot x_{k-1}
\end{array}
$$

Since this algorithm works under the iteration principle, at every iteration it tries to adapt its output sequence to the self realized input sequence. Thus, it's conjointly referred to as self-learning equalizer. The convergence rate and precision to output sequence are the two main design concerns in SATO's blind equalization. To induce the simplest result from Sato's algorithm, the design considerations ought to be optimized on the basis of its parameters, so it'll converge in no time with a high precision output sequence. This could be more or less guided by tap-adjusting coefficient ' $\alpha$ ', as a result of the remaining parameters are not variable in step with [4]. The figure. 3 depicts the structure of SATO primarily based blind equalizer with five taps. In blind approach, the retrieval of each symbol is done with centre tap initialization. The tap coefficient values are calculable victimization (4).

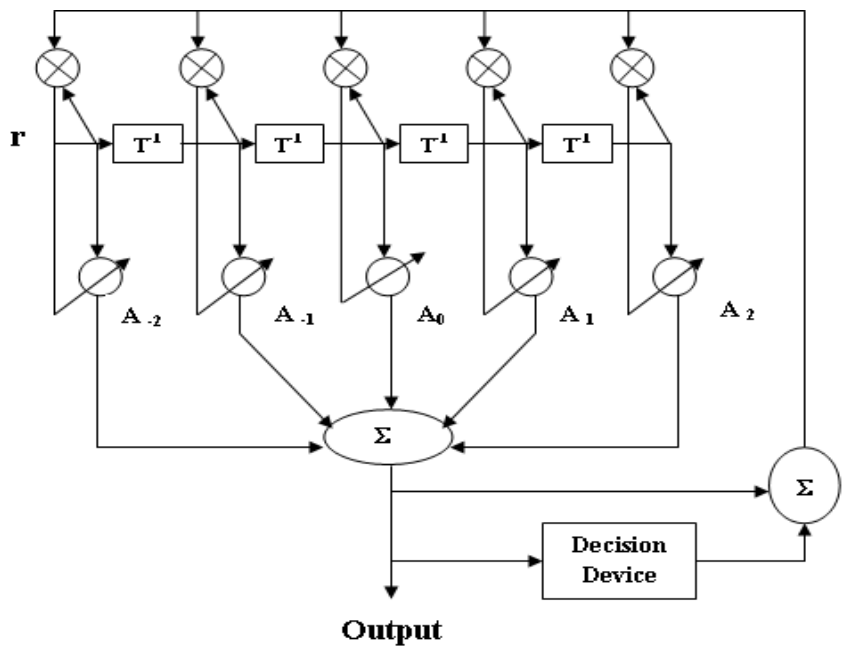

Figure 3: The SATO Based Blind Equalizer with 5 Taps

\section{SimULATION RESUlTS}

We have conducted simulations in finding out the performance of LMS adaptive algorithm and SATO Blind algorithm. For performance analysis, we have a tendency to contemplate the transmission of PAM symbols having ISI with five reflections and AWGN as noise being given as input to the equalizer.

In this approach, the input data sequence was assumed to be independent and drawn from PAM signaling sources. The equalizers are implemented by a linear transversal filter with a five complex tap circuitry. The waveforms shown in Figure 4, Figure 5, Figure 6 and Figure 7 are the results of simulations for received symbol 1 (with ISI and Noise), reconstructed symbol 1 by victimization LMS approach, reconstructed symbol 1 by victimization Blind approach and MSE comparison between LMS and Blind approaches respectively. During this figure. 5 and figure.6 seems identical because both are reconstructed with same SNR 20dB however number of iterations differs. Table 1 shows the quantity of iterations taken by LMS algorithm, with completely different SNR value for the reconstruction of symbol 1,2, 3, 4 and 5 victimization step size parameter $\mu=0.015$ [12].

Table 1. Comparison of SNR vs. Iterations for LMS Adaptive Equalizer with Step Size Parameter $\mu=0.015$

\begin{tabular}{|c|c|c|c|c|c|}
\hline \multirow{2}{*}{$\begin{array}{c}\text { SNR } \\
\text { in } \\
\text { dB }\end{array}$} & \multicolumn{5}{|c|}{ Number of Iterations } \\
\cline { 2 - 6 } & Symbol 1 & Symbol 2 & $\begin{array}{c}\text { Symbol } \\
3\end{array}$ & Symbol 4 & $\begin{array}{c}\text { Symbol } \\
5\end{array}$ \\
\hline 10 & 2 & 1 & 1 & 1 & 1 \\
\hline 12 & 16 & 10 & 7 & 1 & 1 \\
\hline 14 & 36 & 30 & 22 & 13 & 12 \\
\hline 16 & 70 & 75 & 59 & 52 & 52 \\
\hline 18 & 159 & 176 & 154 & 135 & 148 \\
\hline 20 & 362 & 396 & 436 & 333 & 339 \\
\hline
\end{tabular}


Table 2. Comparison of SNR vs. Iterations for SATO based Blind Equalizer with Step Size Parameter $\alpha=.0006$

\begin{tabular}{|c|c|c|c|c|c|}
\hline \multirow{2}{*}{$\begin{array}{c}\text { SNR } \\
\text { in } \\
\text { dB }\end{array}$} & \multicolumn{5}{|c|}{ Number of Iterations } \\
\cline { 2 - 6 } & Symbol 1 & Symbol 2 & Symbol 3 & Symbol 4 & $\begin{array}{c}\text { Symbol } \\
5\end{array}$ \\
\hline 10 & 1123 & 597 & 374 & 111 & 1 \\
\hline 12 & 1643 & 983 & 546 & 180 & 50 \\
\hline 14 & 2406 & 1670 & 849 & 449 & 372 \\
\hline 16 & 3862 & 3019 & 1672 & 1371 & 1218 \\
\hline 18 & 7120 & 5956 & 5194 & 4299 & 4232 \\
\hline 20 & 22908 & 35281 & 35653 & 21378 & 19875 \\
\hline
\end{tabular}

Table 3.Comparison of SNR vs. Iterations for SATO Based Blind Equalizer with Step Size Parameter $\alpha=0.6$

\begin{tabular}{|c|c|c|c|c|c|}
\hline \multirow{2}{*}{$\begin{array}{c}\text { SNR } \\
\text { in } \\
\text { dB }\end{array}$} & \multicolumn{5}{|c|}{ Number of Iterations } \\
\cline { 2 - 6 } & Symbol 1 & Symbol 2 & Symbol 3 & Symbol 4 & $\begin{array}{c}\text { Symbol } \\
5\end{array}$ \\
\hline 10 & 1 & 2 & 2 & 2 & 2 \\
\hline 12 & 2 & 2 & 2 & 2 & 3 \\
\hline 14 & 2 & 3 & 3 & 3 & 3 \\
\hline 16 & 4 & 4 & 3 & 4 & 4 \\
\hline 18 & 7 & 6 & 5 & 5 & 5 \\
\hline 20 & 17 & 23 & 40 & 18 & 33 \\
\hline
\end{tabular}

In this paper, we've used the tap adjusting coefficient value $\left(\alpha=0.6 \times 10^{-3}\right)$, as projected by SATO to reconstruct the PAM signal that is shown in table 2 . For a value of $\alpha=0.6$, we tend to get better convergence as shown in table 3. But, whereas further increasing the value of $\alpha(>0.6)$ ends up in unsuccessful reconstruction of original PAM symbols. The Simulation results show that Sato's Blind algorithm with optimum $\alpha$ value has quicker convergence rate compared to that of LMS algorithm. That is, the quantity of iterations to obtain the same output SNR for identical symbol is much lesser within the Sato primarily based blind approach with $\alpha=$ 0.6 .

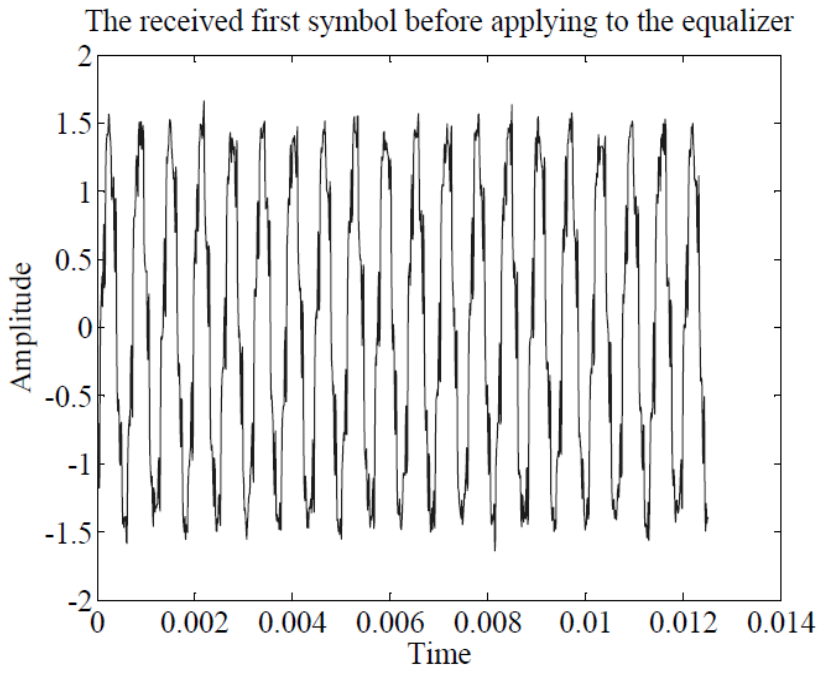

Figure 4: The Received Symbol with ISI and Noise

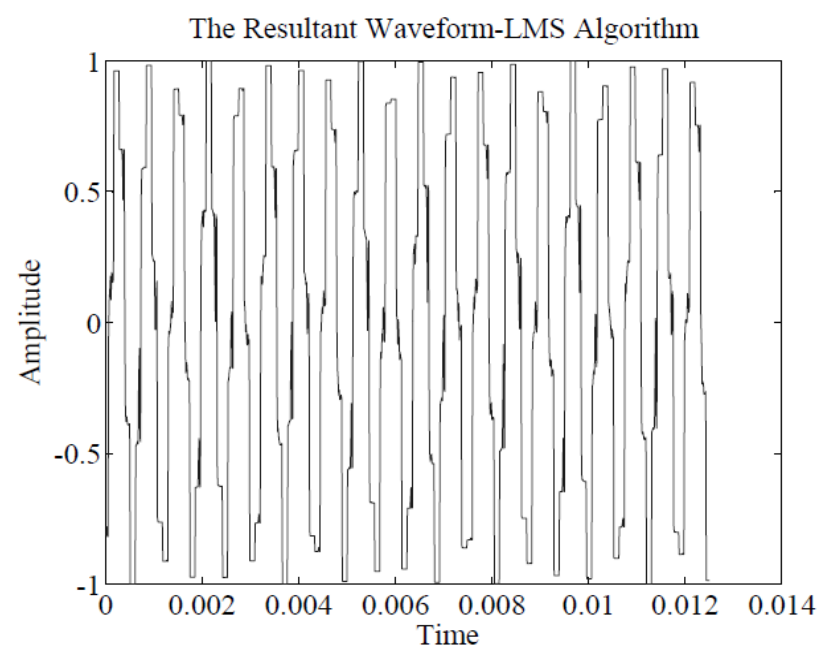

Figure 5: Reconstructed Symbol 1 using LMS Algorithm with $\mu=0.015$ and $\mathrm{SNR}=20 \mathrm{~dB}$ (362 iterations)

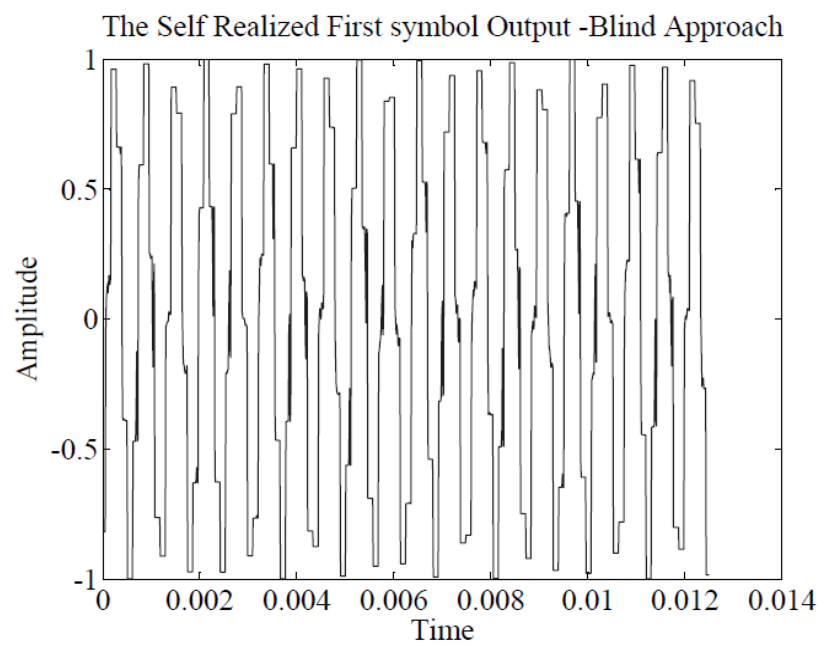


Figure 6: Reconstructed Symbol 1 using SATO Blind algorithm with $\alpha=0.6$ and SNR $=20 \mathrm{~dB}$ (17 iterations)

LMS Vs Blind with optimum alpha Approach

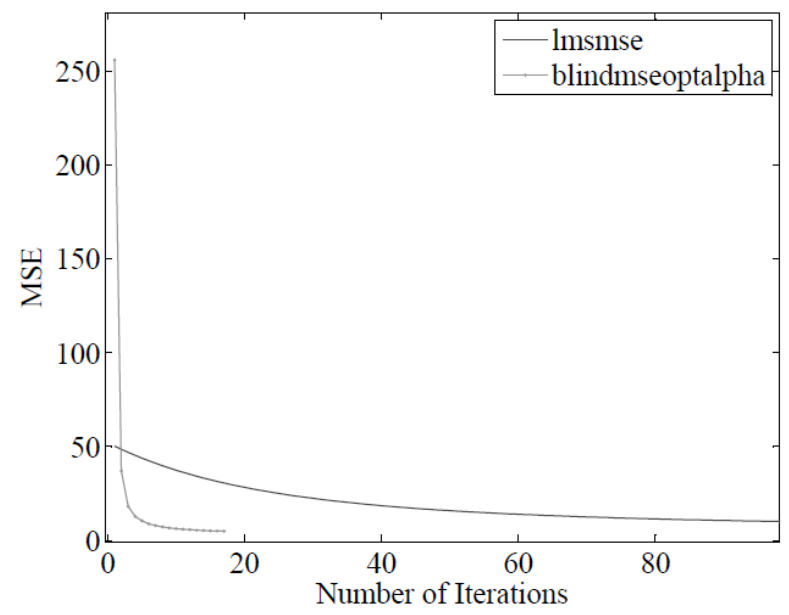

Figure 7:Mean Square Error Comparison between LMS and Blind Approach.

\section{CONCLUSION}

In this paper we compared the performance of LMS adaptive equalizer and SATO based mostly Blind equalizer. Observations from table II and table III show that, the specified SNR will be obtained with less number of iterations in SATO based mostly Blind equalizers by selecting best $\alpha$ value.

Increase in the tap adjusting coefficient value (e.g., $\alpha=0.6$ ) provides a much quicker convergence. When $\alpha=0.7$ some symbols have converged quickly,but some symbols do not converge (due to misadjustment).Similarly for higher values $(\alpha>0.7)$, converge for all PAM symbols does not take place. So, if the optimum value for $\alpha$ may be calculable, the convergence are going to be quick. Rather than a fixed $\alpha$ (this should be unbroken adequately little to create certain stability under all likely operative conditions) value, variable $\alpha$ value for iteration basis will be used to speed up the convergence and minimize the misadjustment.

\section{REFERENCES}

[1] Yoichi Sato, "A Method of Self-Recovering Equalization for Multilevel Amplitude-Modulation Systems", IEEE Transactions on Communications, vol.COM-23, Pp. 679-682, June 1975.

[2] Dominique N. Godard, "Self-Recovering Equalization and Carrier Tracking in Two Dimensional Data Communication Systems”, IEEE Transactions on Communications, vol.COM-28, No.11, Pp. 1867-1875, 1980.

[3] M. Hodges, "The GSM Radio Interface", British Telecom Technological Journal, vol. 8, no. 1, Pp. 31-43, 1990.

[4] S.Guo Yecai, He Longqing and Zhang Yanping, "Design and Implementation of Adaptive Equalizer Based on FPGA", The Eighth International Conference on Electronic Measurement and Instruments (ICEMI,),Pp. 790-794, 2007.

[5] Rappaport Theodore S. "Wireless communications: principles and practice",2nd ed., Pearson Education, 2010.

[6] Kil Nam Oh and Jae Hong Park, "Property Restoral Approach to Blind Equalization of Digital Transmission Channels", IEEE Transactions on Consumer Electronics, vol.43, no.3, Pp.544-549, August 1997.
[7] Ye Li and Zhi Ding, "Convergence Analysis of Finite Length Blind Adaptive Equalizers" IEEE Transactions on Signal Processing, vol. 43, no. 9, Pp. 2120-2129, September 1995.

[8] S.U.H.Qureshi, "Adaptive Equalization", in proc. IEEE, vol. 73, Pp. 1349-1387, September1985.

[9] David Smalley, "Equalization Concepts: A Tutorial", Application Report, Atlanta Regional Technology Centre, SPRA140, October 1994.

[10] A. Benveniste and M. Goursat, "Blind equalizer"IEEE Transactions on Communications, vol. COM-32, Pp.871-883, Aug. 1984.

[11] K. Suthendran, V.R.S.Mani, and V.Vijayarengan, "Design of Blind Equalizer," in Proc. NATCON'06, paper 43, Pp. 206-212, 2006.

[12] R. Perry, David R. Bull, and A. Nix, "Pipelined DFE Architectures using Delayed Coefficient Adaptation" IEEE Transactions on Circuits and Systems-II: Analog and Digital Signal Processing, vol. 45, no. 7,Pp 868-873, July 1998.

[13] David Falconer, "History of equalization 1860-1980" IEEE Communications magazine, Pp 42-50, October 2011. 